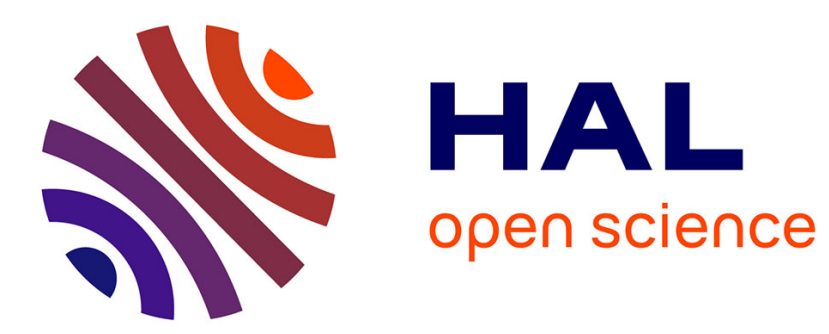

\title{
A note on Spitzer identity for random walk
}

Aimé Lachal

\section{To cite this version:}

Aimé Lachal. A note on Spitzer identity for random walk. Statistics and Probability Letters, 2009, 78 (2), pp.97. 10.1016/j.spl.2007.05.025 . hal-00594447

\section{HAL Id: hal-00594447 https://hal.science/hal-00594447}

Submitted on 20 May 2011

HAL is a multi-disciplinary open access archive for the deposit and dissemination of scientific research documents, whether they are published or not. The documents may come from teaching and research institutions in France or abroad, or from public or private research centers.
L'archive ouverte pluridisciplinaire HAL, est destinée au dépôt et à la diffusion de documents scientifiques de niveau recherche, publiés ou non, émanant des établissements d'enseignement et de recherche français ou étrangers, des laboratoires publics ou privés. 


\section{Author's Accepted Manuscript}

A note on Spitzer identity for random walk

Aimé Lachal

PII:

S0167-7152(07)00200-3

DOI: $\quad$ doi:10.1016/j.spl.2007.05.025

Reference: $\quad$ STAPRO 4680

To appear in: $\quad$ Statistics \& Probability Letters

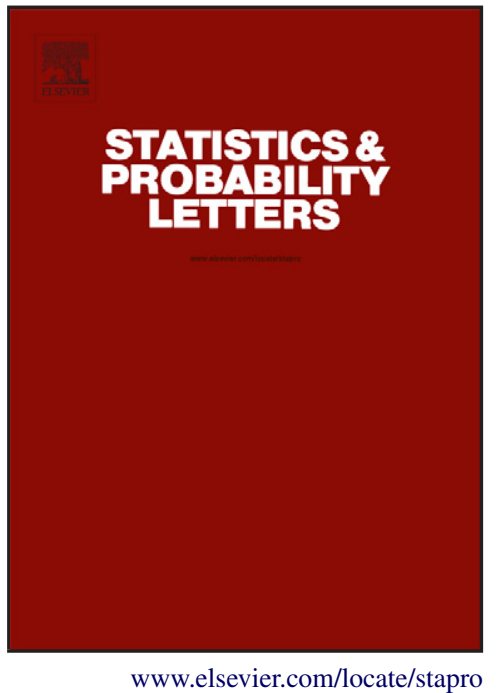

Received date: $\quad 9$ October 2006

Revised date: 16 December 2006

Accepted date: 23 May 2007

Cite this article as: Aimé Lachal, A note on Spitzer identity for random walk, Statistics \& Probability Letters (2007), doi:10.1016/j.spl.2007.05.025

This is a PDF file of an unedited manuscript that has been accepted for publication. As a service to our customers we are providing this early version of the manuscript. The manuscript will undergo copyediting, typesetting, and review of the resulting galley proof before it is published in its final citable form. Please note that during the production process errors may be discovered which could affect the content, and all legal disclaimers that apply to the journal pertain. 


\title{
A note on Spitzer identity for random walk
}

\author{
Aimé LACHAL * \\ Institut National des Sciences Appliquées de Lyon
}

\begin{abstract}
Let $\left(S_{n}\right)_{n \geqslant 0}$ be a random walk evolving on the real line and introduce the first hitting time of the half-line $(a,+\infty)$ for any real $a: \tau_{a}=\min \left\{n \geqslant 1: S_{n}>a\right\}$. The classical Spitzer identity (1960) supplies an expression for the generating function of the couple $\left(\tau_{0}, S_{\tau_{0}}\right)$. In (1998), Nakajima [Kodai Math. J. 21, 192-200] derived a relationship between the generating functions of the random couples $\left(\tau_{0}, S_{\tau_{0}}\right)$ and $\left(\tau_{a}, S_{\tau_{a}}\right)$ for any positive number $a$. In this note, we propose a new and shorter proof for this relationship and complement this analysis by considering the case of an increasing random walk. We especially investigate the Erlangian case and provide an explicit expression for the joint distribution of $\left(\tau_{a}, S_{\tau_{a}}\right)$ in this situation.
\end{abstract}

AMS 2000 SUBJECT CLASSIFICATIONS: PRIMARY 60J15;

SECONDARY 60G50.

Key words and Phrases: Spitzer identity, Laplace-Fourier transform, Erlang distribution.

\section{Introduction}

Let $\left(X_{n}\right)_{n \geqslant 0}$ be a sequence of independent and identically distributed real random variables. Set $S_{0}=0$ and $S_{n}=X_{1}+\cdots+X_{n}$ for $n \geqslant 1$. The sequence $\left(S_{n}\right)_{n \geqslant 0}$ is the usual associated random walk. For any real $a$, we introduce the first hitting time of the half-line $(a,+\infty)$ for the random walk: $\tau_{a}=\min \left\{n \geqslant 1: S_{n}>a\right\}$ (with the usual convention $\min \emptyset=\infty)$ and we put, for $\lambda>0$ and $\mu \in \mathbb{R}$,

$$
\varphi(\lambda, \mu ; a)=\mathbb{E}\left(e^{-\lambda \tau_{a}+i \mu S_{\tau_{a}}} \mathbb{1}_{\left\{\tau_{a}<\infty\right\}}\right)
$$

The function $(\lambda, \mu) \longmapsto \varphi(\lambda, \mu ; a)$ is the Laplace-Fourier transform of the random couple $\left(\tau_{a}, S_{\tau_{a}}\right)$. Let us recall one variant of the famous Spitzer identity corresponding to the case $a=0$ (see, e.g., [1]).

Theorem 1 (Spitzer, 1960) For any $\lambda>0$ and $\mu \in \mathbb{R}$, the following identity holds:

$$
1-\varphi(\lambda, \mu ; 0)=\exp \left(-\sum_{n=1}^{\infty} \frac{e^{-\lambda n}}{n} \mathbb{E}\left(e^{i \mu S_{n}} \mathbb{1}_{\left\{S_{n}>0\right\}}\right)\right) .
$$

*Postal adress: Institut National Des Sciences Appliquées de Lyon Bâtiment Léonard de Vinci, 20 avenue Albert Einstein 69621 Villeurbanne Cedex, France E-mail: aime.lachal@insa-lyon.fr Web page: http://maths.univ-lyon1.fr/ $\sim^{\sim a c h a l}$ 
Remarking that (1) involves only time $\tau_{0}$ which is the first time the random walk overshoots level 0 (when starting therein at time 0), a similar identity concerning the case of any level $a$ would be interesting. In 1998, Nakajima [3] tackled this question and derived a relationship between the Laplace-Fourier transforms of the random couples $\left(\tau_{0}, S_{\tau_{0}}\right)$ and $\left(\tau_{a}, S_{\tau_{a}}\right)$ for any positive number $a$. His result states as follows.

Theorem 2 (Nakajima, 1998) Let $a>0$. For any $\lambda>0$ and $\mu \in \mathbb{R}$, the following identity holds:

$$
1-\varphi(\lambda, \mu ; a)=\lim _{\varepsilon \rightarrow 0^{+}} \int_{-\infty}^{+\infty} \frac{e^{i(a+\varepsilon) \nu}-e^{-i \varepsilon \nu}}{2 \pi i \nu} \frac{1-\varphi(\lambda, \mu ; 0)}{1-\varphi(\lambda, \mu-\nu ; 0)} d \nu
$$

where the integral $\int_{-\infty}^{+\infty}$ stands for a principal value: $\int_{-\infty}^{+\infty}=\lim _{T \rightarrow+\infty} \int_{-T}^{T}$.

Nakajima gave a proof relying on the successive times of increase of the random walk. He introduced several measures related to the random walk and level $a$ and performed a meticulous analysis with. As an illustration, he applied (2) to the case of a two-sided exponential random walk.

Substituting $\lambda=\mu=0$ in (1) and (2), we get the expressions below for the so-called escape or ruin probabilities $\mathbb{P}\left\{\tau_{0}=\infty\right\}$ and $\mathbb{P}\left\{\tau_{a}=\infty\right\}$.

Corollary 1 Let $a>0$. The following identities hold:

$$
\begin{aligned}
& \mathbb{P}\left\{\tau_{0}=\infty\right\}=\exp \left(-\sum_{n=1}^{\infty} \frac{1}{n} \mathbb{P}\left\{S_{n}>0\right\}\right) \\
& \mathbb{P}\left\{\tau_{a}=\infty\right\}=\lim _{\varepsilon \rightarrow 0^{+}} \int_{-\infty}^{+\infty} \frac{e^{i(a+\varepsilon) \nu}-e^{-i \varepsilon \nu}}{2 \pi i \nu} \frac{\mathbb{P}\left\{\tau_{0}=\infty\right\}}{1-\varphi(0,-\nu ; 0)} d \nu .
\end{aligned}
$$

In this note, we revisit this matter and propose an alternative and shorter proof of (2) with concise computations by using the Laplace transform of the function $a \longmapsto \varphi(\lambda, \mu ; a)$ (Section 2). We complement this analysis by computing the integral lying in (2) by residues (Section 3). We next consider the particular case where the random walk is increasing (Section 4) and illustrate this case with an Erlangian situation for which we have explicitly obtained the joint distribution of the random couple $\left(\tau_{a}, S_{\tau_{a}}\right)$ (Subsection 4.3). We finally end up this note by considering the case of a pseudo-random walk (Section 5) which is useful for studying certain continuous-time pseudo-processes driven by some high-order heat-type equations.

\section{Proof of Theorem 2}

As in [3], we introduce the successive times of increase of the random walk $\left(S_{n}\right)_{n \geqslant 0}$ :

$$
\sigma_{0}=0, \quad \sigma_{1}=\min \left\{k \geqslant 1: S_{k}>0\right\}=\tau_{0}
$$

and for $n \geqslant 1$, in a recursive way:

$$
\sigma_{n+1}=\min \left\{k \geqslant \sigma_{n}: S_{k}>S_{\sigma_{n}}\right\} .
$$

If the set $\left\{k \geqslant \sigma_{n}: S_{k}>S_{\sigma_{n}}\right\}$ is empty, we put $\sigma_{n+1}=\infty$. In these settings, the sequence $\left(S_{\sigma_{n}}\right)_{n \geqslant 0}$ is increasing, this is the so-called ladder random walk. 
The probability that time $\sigma_{n}$ be finite is easy to compute by recursion. Although this probability will not be used here, we find it interesting to write it down. We have

$$
\mathbb{P}\left\{\sigma_{n+1}<\infty\right\}=\mathbb{P}\left\{\sigma_{n}<\infty \text { and } \exists k \geqslant \sigma_{n}, S_{k}>S_{\sigma_{n}}\right\} .
$$

Remarking that the events $\left\{\sigma_{n}<\infty\right\}$ and $\left\{\exists k \geqslant \sigma_{n}, S_{k}>S_{\sigma_{n}}\right\}$ are independent, and that this latter has the same probability as the event $\left\{\exists k \geqslant 1, S_{k}>0\right\}$, we get

$$
\mathbb{P}\left\{\sigma_{n+1}<\infty\right\}=\mathbb{P}\left\{\sigma_{n}<\infty\right\} \mathbb{P}\left\{\exists k \geqslant 1, S_{k}>0\right\}=\mathbb{P}\left\{\tau_{0}<\infty\right\} \mathbb{P}\left\{\sigma_{n}<\infty\right\} .
$$

We see that $\left(\mathbb{P}\left\{\sigma_{n}<\infty\right\}\right)_{n \geqslant 1}$ is a geometrical sequence of ratio $\mathbb{P}\left\{\tau_{0}<\infty\right\}$ and then, for $n \geqslant 1$,

$$
\mathbb{P}\left\{\sigma_{n}<\infty\right\}=\mathbb{P}\left\{\tau_{0}<\infty\right\}^{n} .
$$

\section{- Step 1}

By writing $\sigma_{n+1}=\left(\sigma_{n+1}-\sigma_{n}\right)+\sigma_{n}$ and $S_{\sigma_{n+1}}=\left(S_{\sigma_{n+1}}-S_{\sigma_{n}}\right)+S_{\sigma_{n}}$, and next observing that the couples $\left(\sigma_{n+1}-\sigma_{n}, S_{\sigma_{n+1}}-S_{\sigma_{n}}\right)$ and $\left(\sigma_{1}, S_{\sigma_{1}}\right)$ have identical distributions and that the couples $\left(\sigma_{n+1}-\sigma_{n}, S_{\sigma_{n+1}}-S_{\sigma_{n}}\right)$ and $\left(\sigma_{n}, S_{\sigma_{n}}\right)$ are independent, we first obtain

$$
\mathbb{E}\left(e^{-\lambda \sigma_{n+1}+i \mu S_{\sigma_{n+1}}} \mathbb{1}_{\left\{\sigma_{n+1}<\infty\right\}}\right)=\mathbb{E}\left(e^{-\lambda \sigma_{1}+i \mu S_{\sigma_{1}}} \mathbb{1}_{\left\{\sigma_{1}<\infty\right\}}\right) \times \mathbb{E}\left(e^{-\lambda \sigma_{n}+i \mu S_{\sigma_{n}}} \mathbb{1}_{\left\{\sigma_{n}<\infty\right\}}\right) .
$$

So, we are dealing with a geometrical sequence of ratio $\varphi(\lambda, \mu ; 0)$ and then, for any $n \geqslant 0$,

$$
\mathbb{E}\left(e^{-\lambda \sigma_{n}+i \mu S_{\sigma_{n}}} \mathbb{1}_{\left\{\sigma_{n}<\infty\right\}}\right)=\varphi(\lambda, \mu ; 0)^{n} .
$$

We deduce the relation

$$
\sum_{n=0}^{\infty} \mathbb{E}\left(e^{-\lambda \sigma_{n}+i \mu S_{\sigma_{n}}} \mathbb{1}_{\left\{\sigma_{n}<\infty\right\}}\right)=\frac{1}{1-\varphi(\lambda, \mu ; 0)}
$$

\section{- Step 2}

Second, in the foregoing settings, time $\tau_{a}$ is the instant $\sigma_{n}$ characterized by the double inequality $S_{\sigma_{n-1}} \leqslant a<S_{\sigma_{n}}$; and then $S_{\tau_{a}}=S_{\sigma_{n}}$. Therefore, we can evaluate $\varphi(\lambda, \mu ; a)$ as follows:

$$
\begin{aligned}
\varphi(\lambda, \mu ; a) & =\sum_{n=1}^{\infty} \mathbb{E}\left(e^{-\lambda \sigma_{n}+i \mu S_{\sigma_{n}}} \mathbb{1}_{\left\{\sigma_{n}<\infty, S_{\sigma_{n-1}} \leqslant a<S_{\sigma_{n}}\right\}}\right) \\
& =\sum_{n=1}^{\infty}\left[\mathbb{E}\left(e^{-\lambda \sigma_{n}+i \mu S_{\sigma_{n}}} \mathbb{1}_{\left\{\sigma_{n}<\infty, S_{\sigma_{n}}>a\right\}}\right)-\mathbb{E}\left(e^{-\lambda \sigma_{n}+i \mu S_{\sigma_{n}}} \mathbb{1}_{\left\{\sigma_{n}<\infty, S_{\sigma_{n-1}}>a\right\}}\right)\right] \\
& =\sum_{n=0}^{\infty}\left[\mathbb{E}\left(e^{-\lambda \sigma_{n}+i \mu S_{\sigma_{n}}} \mathbb{1}_{\left\{\sigma_{n}<\infty, S_{\sigma_{n}}>a\right\}}\right)-\mathbb{E}\left(e^{-\lambda \sigma_{n+1}+i \mu S_{\sigma_{n+1}}} \mathbb{1}_{\left\{\sigma_{n+1}<\infty, S_{\sigma_{n}}>a\right\}}\right)\right] .
\end{aligned}
$$

Invoking the previous arguments on the increment $\sigma_{n+1}-\sigma_{n}$, we get

$$
\begin{aligned}
\mathbb{E}\left(e^{-\lambda \sigma_{n+1}+i \mu S_{\sigma_{n+1}}}\right. & \left.\mathbb{1}_{\left\{\sigma_{n+1}<\infty, S_{\sigma_{n}}>a\right\}}\right) \\
= & \mathbb{E}\left(e^{-\lambda \sigma_{n}+i \mu S_{\sigma_{n}}} \mathbb{1}_{\left\{\sigma_{n}<\infty, S_{\sigma_{n}}>a\right\}}\right) \times \mathbb{E}\left(e^{-\lambda \sigma_{1}+i \mu S_{\sigma_{1}}} \mathbb{1}_{\left\{\sigma_{1}<\infty\right\}}\right)
\end{aligned}
$$

and thus, by (4),

$$
\begin{aligned}
\varphi(\lambda, \mu ; a) & =[1-\varphi(\lambda, \mu ; 0)] \sum_{n=1}^{\infty} \mathbb{E}\left(e^{-\lambda \sigma_{n}+i \mu S_{\sigma_{n}}} \mathbb{1}_{\left\{\sigma_{n}<\infty, S_{\sigma_{n}}>a\right\}}\right) \\
& =[1-\varphi(\lambda, \mu ; 0)] \sum_{n=0}^{\infty}\left[\mathbb{E}\left(e^{-\lambda \sigma_{n}+i \mu S_{\sigma_{n}}} \mathbb{1}_{\left\{\sigma_{n}<\infty\right\}}\right)-\mathbb{E}\left(e^{-\lambda \sigma_{n}+i \mu S_{\sigma_{n}}} \mathbb{1}_{\left\{\sigma_{n}<\infty, S_{\sigma_{n}} \leqslant a\right\}}\right)\right] \\
& =1-[1-\varphi(\lambda, \mu ; 0)] \sum_{n=0}^{\infty} \mathbb{E}\left(e^{-\lambda \sigma_{n}+i \mu S_{\sigma_{n}}} \mathbb{1}_{\left\{\sigma_{n}<\infty, S_{\sigma_{n}} \leqslant a\right\}}\right) .
\end{aligned}
$$


Putting

$$
\psi(\lambda, \mu ; a)=\sum_{n=0}^{\infty} \mathbb{E}\left(e^{-\lambda \sigma_{n}+i \mu S_{\sigma_{n}}} \mathbb{1}_{\left\{\sigma_{n}<\infty, S_{\sigma_{n}} \leqslant a\right\}}\right),
$$

we have obtained the relation

$$
\psi(\lambda, \mu ; a)=\frac{1-\varphi(\lambda, \mu ; a)}{1-\varphi(\lambda, \mu ; 0)}
$$

\section{- Step 3}

We now take the Laplace transform with respect to $a$ of (5). By (3), we find that

$$
\begin{aligned}
\int_{0}^{+\infty} e^{-\nu a} \psi(\lambda, \mu ; a) d a & =\sum_{n=0}^{\infty} \mathbb{E}\left(e^{-\lambda \sigma_{n}+i \mu S_{\sigma_{n}}} \int_{0}^{+\infty} e^{-\nu a} \mathbb{1}_{\left\{\sigma_{n}<\infty, S_{\sigma_{n}} \leqslant a\right\}} d a\right) \\
& =\frac{1}{\nu} \sum_{n=0}^{\infty} \mathbb{E}\left(e^{-\lambda \sigma_{n}+(i \mu-\nu) S_{\sigma_{n}}} \mathbb{1}_{\left\{\sigma_{n}<\infty\right\}}\right) \\
& =\frac{1}{\nu} \sum_{n=0}^{\infty} \varphi(\lambda, \mu+i \nu ; 0)^{n}=\frac{1}{\nu[1-\varphi(\lambda, \mu+i \nu ; 0)]}
\end{aligned}
$$

The classical inversion formula yields for any $c>0$ (see, e.g., [5, Theorem 7.3, p. 66])

$$
\int_{c-i \infty}^{c+i \infty} \frac{1}{2 \pi i} \frac{e^{b \nu}}{\nu[1-\varphi(\lambda, \mu+i \nu ; 0)]} d \nu= \begin{cases}\frac{1}{2}\left[\psi\left(\lambda, \mu ; b^{+}\right)+\psi\left(\lambda, \mu ; b^{-}\right)\right] & \text {if } b>0 \\ \frac{1}{2} \psi\left(\lambda, \mu ; 0^{+}\right) & \text {if } b=0 \\ 0 & \text { if } b<0\end{cases}
$$

where the integral $\int_{c-i \infty}^{c+i \infty}$ stands for a principal value. On the other hand, it may be easily seen that the function $a \longmapsto \psi(\lambda, \mu ; a)$ is right-continuous, so the foregoing equality supplies, by replacing $b$ by $a+\varepsilon$ therein and next taking the limit as $\varepsilon$ tends to $0^{+}$:

$$
\psi(\lambda, \mu ; a)=\lim _{\varepsilon \rightarrow 0^{+}} \int_{c-i \infty}^{c+i \infty} \frac{e^{(a+\varepsilon) \nu}}{2 \pi i \nu[1-\varphi(\lambda, \mu+i \nu ; 0)]} d \nu
$$

and we also have by replacing $b$ by $-\varepsilon$

$$
\lim _{\varepsilon \rightarrow 0^{+}} \int_{c-i \infty}^{c+i \infty} \frac{e^{-\varepsilon \nu}}{2 \pi i \nu[1-\varphi(\lambda, \mu+i \nu ; 0)]} d \nu=0
$$

Subtracting (8) from (7), we get

$$
\psi(\lambda, \mu ; a)=\lim _{\varepsilon \rightarrow 0^{+}} \int_{c-i \infty}^{c+i \infty} \frac{e^{(a+\varepsilon) \nu}-e^{-\varepsilon \nu}}{2 \pi i \nu[1-\varphi(\lambda, \mu+i \nu ; 0)]} d \nu .
$$

Now, since the function $\left[e^{(a+\varepsilon) \nu}-e^{-\varepsilon \nu}\right] / \nu$ has no singularity at $\nu=0$, we can let $c$ tend to $0^{+}$in (9) and by plugging the corresponding result into (6), (2) immediately emerges.

Remark 1 As a check, we can conversely write, using (4),

$$
\begin{aligned}
\int_{-\infty}^{+\infty} \frac{e^{i(a+\varepsilon) \nu}-e^{-i \varepsilon \nu}}{2 \pi i \nu} & \frac{d \nu}{1-\varphi(\lambda, \mu-\nu ; 0)} \\
= & \sum_{n=0}^{\infty} \int_{-\infty}^{+\infty} \frac{e^{i(a+\varepsilon) \nu}-e^{-i \varepsilon \nu}}{2 \pi i \nu} \mathbb{E}\left(e^{-\lambda \sigma_{n}+i(\mu-\nu) S_{\sigma_{n}}}\right) d \nu \\
= & \sum_{n=0}^{\infty} \mathbb{E}\left(e^{-\lambda \sigma_{n}+i \mu S_{\sigma_{n}}} \int_{-\infty}^{+\infty} \frac{e^{i\left(a+\varepsilon-S_{\sigma_{n}}\right) \nu}-e^{-i\left(\varepsilon+S_{\sigma_{n}}\right) \nu}}{2 \pi i \nu} d \nu\right) .
\end{aligned}
$$


For the convenience of the reader, we recall the value of Dirichlet integral: for $a<b$,

$$
\int_{-\infty}^{+\infty} \frac{e^{i b \nu}-e^{i a \nu}}{2 \pi i \nu} d \nu= \begin{cases}1 & \text { if } a<0<b \\ 1 / 2 & \text { if } a=0<b \text { or } a<b=0 \\ 0 & \text { if } a, b<0 \text { or } a, b>0\end{cases}
$$

With this result at hand and reminding that $S_{\sigma_{n}} \geqslant 0$, we get

$$
\begin{aligned}
& \int_{-\infty}^{+\infty} \frac{e^{i(a+\varepsilon) \nu}-e^{-i \varepsilon \nu}}{2 \pi i \nu} \frac{d \nu}{1-\varphi(\lambda, \mu-\nu ; 0)} \\
& \quad=\sum_{n=0}^{\infty} \mathbb{E}\left(e^{-\lambda \sigma_{n}+i \mu S_{\sigma_{n}}} \mathbb{1}_{\left\{\sigma_{n}<\infty, S_{\sigma_{n}}<a+\varepsilon\right\}}\right)+\frac{1}{2} \sum_{n=0}^{\infty} \mathbb{E}\left(e^{-\lambda \sigma_{n}+i \mu S_{\sigma_{n}}} \mathbb{1}_{\left\{\sigma_{n}<\infty, S_{\sigma_{n}}=a+\varepsilon\right\}}\right) .
\end{aligned}
$$

On the other hand, we plainly have

$$
\mathbb{P}\left\{\sigma_{n}<\infty, S_{\sigma_{n}}=a+\varepsilon\right\}=\mathbb{P}\left\{\sigma_{n}<\infty, S_{\sigma_{n}} \leqslant a+\varepsilon\right\}-\mathbb{P}\left\{\sigma_{n}<\infty, S_{\sigma_{n}}<a+\varepsilon\right\}
$$

and, because of $\cap_{\varepsilon>0}(-\infty, a+\varepsilon]=\cap_{\varepsilon>0}(-\infty, a+\varepsilon)=(-\infty, a]$, this yields

$$
\lim _{\varepsilon \rightarrow 0^{+}} \mathbb{P}\left\{\sigma_{n}<\infty, S_{\sigma_{n}} \leqslant a+\varepsilon\right\}=\lim _{\varepsilon \rightarrow 0^{+}} \mathbb{P}\left\{\sigma_{n}<\infty, S_{\sigma_{n}}<a+\varepsilon\right\}=\mathbb{P}\left\{\sigma_{n}<\infty, S_{\sigma_{n}} \leqslant a\right\},
$$

from which we extract $\lim _{\varepsilon \rightarrow 0^{+}} \mathbb{P}\left\{\sigma_{n}<\infty, S_{\sigma_{n}}=a+\varepsilon\right\}=0$. As a result, by (5) and (6), (10) becomes

$$
\lim _{\varepsilon \rightarrow 0^{+}} \int_{-\infty}^{+\infty} \frac{e^{i(a+\varepsilon) \nu}-e^{-i \varepsilon \nu}}{2 \pi i \nu} \frac{d \nu}{1-\varphi(\lambda, \mu-\nu ; 0)}=\psi(\lambda, \mu ; a)=\frac{1-\varphi(\lambda, \mu ; a)}{1-\varphi(\lambda, \mu ; 0)} .
$$

This computation ascertains (2).

Remark 2 It could be tempting to replace the limit lying in (2) by the simpler expression (without any limit) $\int_{-\infty}^{+\infty} \frac{e^{i a \nu}-1}{2 \pi i \nu} \frac{1-\varphi(\lambda, \mu ; 0)}{1-\varphi(\lambda, \mu-\nu ; 0)} d \nu$. Actually, this can be wrong as shows the example below concerning the increasing Bernoulli random walk evolving on the lattice $\{k b, k \geqslant 0\}$ for which the hitting level of interest is $a=n b$ for an certain positive integer $n$.

Let us choose for the common distribution of the $X_{n}$ 's the Bernoulli law of parameter $1-p$ and support $\{0, b\}$ where $b$ is a fixed positive number: $\mathbb{P}\left\{X_{n}=0\right\}=p$ and $\mathbb{P}\left\{X_{n}=b\right\}$ $=1-p$. Then the distribution of $S_{n}$ is the classical Binomial law of parameters $(n, 1-p)$ on the set $\{k b, 0 \leqslant k \leqslant n\}$. Since the $X_{n}$ 's are non negative for all $n \geqslant 0$, the sequence $\left(S_{n}\right)_{n \geqslant 0}$ is non decreasing. Thus, referring to Section 4.2 , we get

$$
\frac{1-\varphi(\lambda, \mu ; 0)}{1-\varphi(\lambda, \mu-\nu ; 0)}=\frac{1-e^{-\lambda}\left(p+(1-p) e^{i b \mu}\right)}{1-e^{-\lambda}\left(p+(1-p) e^{i b(\mu-\nu)}\right)}=\frac{1-A}{1-A e^{-i b \nu}}=\sum_{k=0}^{\infty}(1-A) A^{k} e^{-i k b \nu}
$$

where we set $A=\frac{1-p}{e^{\lambda}-p} e^{i b \mu}$. We have

$$
\begin{aligned}
\lim _{\varepsilon \rightarrow 0^{+}} \int_{-\infty}^{+\infty} \frac{e^{i(a+\varepsilon) \nu}-e^{-i \varepsilon \nu}}{2 \pi i \nu} \frac{1-\varphi(\lambda, \mu ; 0)}{1-\varphi(\lambda, \mu-\nu ; 0)} d \nu \\
=\sum_{k=0}^{\infty}(1-A) A^{k} \lim _{\varepsilon \rightarrow 0^{+}} \int_{-\infty}^{+\infty} \frac{e^{i(a-k b+\varepsilon) \nu}-e^{-i(k b+\varepsilon) \nu}}{2 \pi i \nu} d \nu
\end{aligned}
$$

By Dirichlet integral, we see that

$$
\lim _{\varepsilon \rightarrow 0^{+}} \int_{-\infty}^{+\infty} \frac{e^{i(a-k b+\varepsilon) \nu}-e^{-i(k b+\varepsilon) \nu}}{2 \pi i \nu} d \nu= \begin{cases}1 & \text { if } k \leqslant a / b, \\ 0 & \text { if } k>a / b\end{cases}
$$


and then, introducing the integer part of $a / b, n_{0}=\lfloor a / b\rfloor$ say,

$$
\lim _{\varepsilon \rightarrow 0^{+}} \int_{-\infty}^{+\infty} \frac{e^{i(a+\varepsilon) \nu}-e^{-i \varepsilon \nu}}{2 \pi i \nu} \frac{1-\varphi(\lambda, \mu ; 0)}{1-\varphi(\lambda, \mu-\nu ; 0)} d \nu=\sum_{k=0}^{n_{0}}(1-A) A^{k}=1-A^{n_{0}+1} .
$$

We finally obtain by $(2)$ that $\varphi(\lambda, \mu ; a)=A^{n_{0}+1}$, that is

$$
\varphi(\lambda, \mu ; a)=\left(\frac{1-p}{e^{\lambda}-p}\right)^{\lfloor a / b\rfloor+1} e^{i(\lfloor a / b\rfloor+1) b \mu} .
$$

Now, if we omitted the term $\varepsilon$ (and then the related limit) in the integral lying in (2), we would get

$$
\int_{-\infty}^{+\infty} \frac{e^{i(a-k b) \nu}-e^{-i(k b) \nu}}{2 \pi i \nu} d \nu= \begin{cases}1 & \text { if } 1 \leqslant k<a / b \\ 1 / 2 & \text { if } k=0 \text { or } k=a / b \text { when being an integer } \\ 0 & \text { if } k>a / b\end{cases}
$$

which would yields the wrong result $(1-A)\left(\frac{1}{2}+\sum_{k=1}^{n_{0}-1} A^{k}+\frac{1}{2} A^{n_{0}}\right)$ for $1-\varphi(\lambda, \mu ; a)$ when $a$ is a multiple of $b$.

From (11), we can immediately see that $S_{\tau_{a}}=(\lfloor a / b\rfloor+1) b$, a predictable fact since the random walk passes through all levels $n b, n \geqslant 0$, in a non-decreasing manner; so it crosses level $a$ by straddling levels $\lfloor a / b\rfloor b$ and $(\lfloor a / b\rfloor+1) b$. On the other hand, we also deduce that

$$
\mathbb{E}\left(e^{-\lambda \tau_{a}}\right)=\left(\frac{1-p}{e^{\lambda}-p}\right)^{\lfloor a / b\rfloor+1}
$$

and we recognize for the distribution of $\tau_{a}$ the Pascal law of parameters $(\lfloor a / b\rfloor, 1-p)$, a well-known distribution in gambling theory. Indeed, imagine the $X_{n}$ 's model a sequence of head or tail gambles when tossing a coin. We say that $X_{n}=0$ if head occurs at the $n$-th gamble, else $X_{n}=b$. The quantity $b$ could be viewed as a (positive) gain. Then $S_{n}$ represents the total gain won during the first $n$ gambles. In this context, $\tau_{a}$ is the minimal number of gambles for getting at least the gain equal to the smallest multiple of $b$ greater than $a$, that is $(\lfloor a / b\rfloor+1) b$.

\section{Computation by residues}

In this section, we assume for simplifying the subsequent calculations that the complex roots of the equation $\varphi(\lambda, \mu ; 0)=1$ with unknown $\mu$ are single and we call them $\mu_{k}, k \in K$. We also assume that $K$ is finite. Observe that, since the random variables $X_{n}$ are real, the opposite of the conjugates $-\bar{\mu}_{k}$ are also roots. Moreover, due to the inequality holding for $\lambda>0$ and $\mu \in \mathbb{R}$ :

$$
|1-\varphi(\lambda, \mu ; a)| \geqslant 1-e^{-\lambda}>0,
$$

we see that the roots are not real. Set $K^{+}=\left\{k \in K: \Im\left(\mu_{k}\right)>0\right\}$ and $K^{-}=\{k \in K$ : $\left.\Im\left(\mu_{k}\right)<0\right\}$. The roots of the equation $\varphi(\lambda, \mu-\nu ; 0)=1$ with unknown $\nu$ are $\mu-\mu_{k}, k \in K$.

We now integrate the functions $\nu \longmapsto \frac{e^{i(a+\varepsilon) \nu}}{\nu[1-\varphi(\lambda, \mu-\nu ; 0)]}$ and $\nu \longmapsto \frac{e^{-i \varepsilon \nu}}{\nu[1-\varphi(\lambda, \mu-\nu ; 0)]}$ on the respective close curves $C^{+}=I_{\alpha, R} \cup C_{\alpha}^{+} \cup C_{R}^{+}$and $C^{-}=I_{\alpha, R} \cup C_{\alpha}^{-} \cup C_{R}^{-}$where, for any $\alpha, r, R>0$ with $\alpha<R$,

$$
I_{\alpha, R}=[-R,-\alpha] \cup[\alpha, R], \quad C_{r}^{+}=\left\{r e^{i \theta}, \theta \in[0, \pi]\right\}, \quad C_{r}^{-}=\left\{r e^{i \theta}, \theta \in[-\pi, 0]\right\} .
$$


We shall let $R$ tend to $+\infty$ and $\alpha$ to $0^{+}$. Applying the theorem of the residues and assuming that the integrals over $C_{R}^{+}$and $C_{R}^{-}$tend to 0 , we easily obtain

$$
\begin{gathered}
\lim _{R \rightarrow+\infty}\left(\int_{-R}^{-\alpha}+\int_{\alpha}^{R}\right) \frac{e^{i(a+\varepsilon) \nu} d \nu}{\nu[1-\varphi(\lambda, \mu-\nu ; 0)]}+\int_{C_{\alpha}^{+}} \frac{e^{i(a+\varepsilon) \nu} d \nu}{\nu[1-\varphi(\lambda, \mu-\nu ; 0)]} \\
=2 \pi i \sum_{k \in K^{+}} \frac{e^{i(a+\varepsilon)\left(\mu-\mu_{k}\right)}}{\left(\mu-\mu_{k}\right) \partial_{\mu} \varphi\left(\lambda, \mu_{k} ; 0\right)} \\
\lim _{R \rightarrow+\infty}\left(\int_{-R}^{-\alpha}+\int_{\alpha}^{R}\right) \frac{e^{-i \varepsilon \nu} d \nu}{\nu[1-\varphi(\lambda, \mu-\nu ; 0)]}+\int_{C_{\alpha}^{-}} \frac{e^{-i \varepsilon \nu} d \nu}{\nu[1-\varphi(\lambda, \mu-\nu ; 0)]} \\
=-2 \pi i \sum_{k \in K^{-}} \frac{e^{-i \varepsilon\left(\mu-\mu_{k}\right)}}{\left(\mu-\mu_{k}\right) \partial_{\mu} \varphi\left(\lambda, \mu_{k} ; 0\right)}
\end{gathered}
$$

For lightening the notations, we have written $\partial_{\mu} \varphi$ for $\frac{\partial \varphi}{\partial \mu}$ in (12) and (13). Moreover, the semi-circles $C_{\alpha}^{+}$and $C_{\alpha}^{-}$in the above integrals are oriented so that the corresponding angles vary respectively from $\pi$ to 0 , and from $-\pi$ to 0 . We intend to subtract (13) from (12) and next to let $\alpha$ tend to $0^{+}$. For this, we plainly see that

$$
\begin{aligned}
& \lim _{\alpha \rightarrow 0^{+}, R \rightarrow+\infty}\left(\int_{-R}^{-\alpha}+\int_{\alpha}^{R}\right)\left(\frac{e^{i(a+\varepsilon) \nu}}{\nu[1-\varphi(\lambda, \mu-\nu ; 0)]}-\frac{e^{-i \varepsilon \nu}}{\nu[1-\varphi(\lambda, \mu-\nu ; 0)]}\right) d \nu \\
&=\int_{-\infty}^{+\infty} \frac{e^{i(a+\varepsilon) \nu}-e^{-i \varepsilon \nu}}{\nu[1-\varphi(\lambda, \mu-\nu ; 0)]} d \nu
\end{aligned}
$$

and that

$$
\lim _{\alpha \rightarrow 0^{+}}\left(\int_{C_{\alpha}^{+}} \frac{e^{i(a+\varepsilon) \nu} d \nu}{\nu[1-\varphi(\lambda, \mu-\nu ; 0)]}-\int_{C_{\alpha}^{-}} \frac{e^{-i \varepsilon \nu} d \nu}{\nu[1-\varphi(\lambda, \mu-\nu ; 0)]}\right)=-\frac{2 \pi i}{1-\varphi(\lambda, \mu ; 0)} .
$$

Now, subtracting (13) from (12) and next letting $\alpha$ and $\varepsilon$ tend to $0^{+}$, expression (14) below entails.

Proposition 1 If the roots of the equation $\varphi(\lambda, \mu ; 0)=1$ with unknown $\mu$ are single and are denoted by $\mu_{k}, k \in K$ where $K$ is finite, if the conditions $\lim _{R \rightarrow+\infty} \int_{0}^{\pi} \frac{e^{i(a+\varepsilon) R e^{i \theta}}}{1-\varphi\left(\lambda, \mu-R e^{i \theta} ; 0\right)} d \theta$ $=\lim _{R \rightarrow+\infty} \int_{-\pi}^{0} \frac{e^{-i \varepsilon R e^{i \theta}}}{1-\varphi\left(\lambda, \mu-R e^{i \theta} ; 0\right)} d \theta=0$ are fulfilled for any sufficiently small $\varepsilon>0$, we have

$$
\begin{aligned}
\varphi(\lambda, \mu ; a)= & {[\varphi(\lambda, \mu ; 0)-1]\left(\sum_{\Im \mu_{k}<0} \frac{e^{i a\left(\mu-\mu_{k}\right)}}{\left(\mu-\mu_{k}\right) \partial_{\mu} \varphi\left(\lambda, \mu_{k} ; 0\right)}\right.} \\
& \left.+\sum_{\Im \mu_{k}>0} \frac{1}{\left(\mu-\mu_{k}\right) \partial_{\mu} \varphi\left(\lambda, \mu_{k} ; 0\right)}\right) .
\end{aligned}
$$

Remark 3 If the number of roots of the equation is infinite, (14) may be wrong and should be replaced by

$$
\begin{aligned}
\varphi(\lambda, \mu ; a)= & {[\varphi(\lambda, \mu ; 0)-1] \lim _{\varepsilon \rightarrow 0^{+}}\left(\sum_{\Im \mu_{k}<0} \frac{e^{i(a+\varepsilon)\left(\mu-\mu_{k}\right)}}{\left(\mu-\mu_{k}\right) \partial_{\mu} \varphi\left(\lambda, \mu_{k} ; 0\right)}\right.} \\
& \left.+\sum_{\Im \mu_{k}>0} \frac{e^{-i \varepsilon\left(\mu-\mu_{k}\right)}}{\left(\mu-\mu_{k}\right) \partial_{\mu} \varphi\left(\lambda, \mu_{k} ; 0\right)}\right) .
\end{aligned}
$$


Indeed, consider again the example of the Bernoulli random walk described in Remark 2. The roots of the equation $\varphi(\lambda, \mu ; 0)=1$ are $\mu_{k}=-\frac{i}{b} \ln \frac{e^{\lambda}-p}{1-p}+\frac{2 k \pi}{b}, k \in K=\mathbb{Z}$. We have $\partial_{\mu} \varphi\left(\lambda, \mu_{k} ; 0\right)=i b$ and

$$
\sum_{\Im \mu_{k}<0} \frac{e^{i(a+\varepsilon)\left(\mu-\mu_{k}\right)}}{\left(\mu-\mu_{k}\right) \partial_{\mu} \varphi\left(\lambda, \mu_{k} ; 0\right)}=i e^{i a \mu-\frac{a+\varepsilon}{b} \ln \frac{e^{\lambda}-p}{1-p}} \sum_{k \in \mathbb{Z}} \frac{e^{-i \frac{a+\varepsilon}{b} 2 k \pi}}{2 k \pi-\left(b \mu+i \ln \frac{e^{\lambda}-p}{1-p}\right)} .
$$

The foregoing sum can be evaluated by using Fourier series. In fact, let us fix a non null complex $\beta$ and let us introduce the 1-periodic function defined by $f(x)=e^{i \beta x}$ for $x \in(0,1)$ that is, explicitly, $f(x)=e^{i \beta(x-\lfloor x\rfloor)}$ for $x \in \mathbb{R} \backslash \mathbb{Z}$. Dirichlet theorem for Fourier series asserts that

$$
\sum_{k \in \mathbb{Z}} \frac{e^{i 2 \pi k x}}{2 \pi k-\beta}= \begin{cases}\frac{i}{1-e^{i \beta}} e^{i \beta(x-\lfloor x\rfloor)} & \text { if } x \in \mathbb{R} \backslash \mathbb{Z}, \\ \frac{i}{2} \frac{1+e^{i \beta}}{1-e^{i \beta}} & \text { if } x \in \mathbb{Z} .\end{cases}
$$

Applying (17) to $x=-(a+\varepsilon) / b$ and $\beta=b \mu+i \ln \frac{e^{\lambda}-p}{1-p}$ and observing that this $x$ is never an integer for any small enough $\varepsilon>0$ (so, we use the first line of (17)), we see that (16) simplifies into

$$
\frac{e^{\left(\left\lfloor\frac{a+\varepsilon}{b}\right\rfloor+1\right)\left(i b \mu-\ln \frac{e^{\lambda}-p}{1-p}\right)}}{\frac{1-p}{e^{\lambda}-p} e^{i b \mu}-1}=\frac{e^{\left(n_{0}+1\right)\left(i b \mu-\ln \frac{e^{\lambda}-p}{1-p}\right)}}{\varphi(\lambda, \mu ; 0)-1}
$$

with $n_{0}=\left\lfloor\frac{a+\varepsilon}{b}\right\rfloor=\left\lfloor\frac{a}{b}\right\rfloor$ for small enough $\varepsilon>0$. Plugging this in (15), we retrieve (11). Now, if we directly apply (14) (without $\varepsilon$ ) in the particular case where $a / b$ is an integer, the second line of (17) produces a wrong term in (16).

\section{The increasing random walk}

In this part, we focus ourselves on the case where all the random variables $X_{n}$ are nonnegative: for all $n \geqslant 0, X_{n} \geqslant 0$. In this case, the random walk $\left(S_{n}\right)_{n \geqslant 0}$ is non-decreasing. Since the $X_{n}$ 's are identically distributed, we shall write $X$ for the generic random variable (not depending on the index $n$ ) which has the common distribution.

Identity (14) takes the form, under the assumptions of Proposition 1,

$$
\varphi(\lambda, \mu ; a)=[\varphi(\lambda, \mu ; 0)-1]\left(\sum_{\Im \mu_{k}<0} \frac{e^{i a\left(\mu-\mu_{k}\right)}}{\left(\mu-\mu_{k}\right) \partial_{\mu} \varphi\left(\lambda, \mu_{k} ; 0\right)}\right) .
$$

Here, we could work with the Laplace transform with respect to $\mu$ instead. Put, for $\lambda>0$ and $\mu>0$,

$$
\tilde{\varphi}(\lambda, \mu ; a)=\mathbb{E}\left(e^{-\lambda \tau_{a}-\mu S_{\tau_{a}}}\right)
$$

and let $\tilde{\mu}_{k}=-i \mu_{k}$ be the roots of the equation $\tilde{\varphi}(\lambda, \mu ; 0)=1$ with unknown $\mu$. Relation (18) writes

$$
\tilde{\varphi}(\lambda, \mu ; a)=[\tilde{\varphi}(\lambda, \mu ; 0)-1]\left(\sum_{\Re \tilde{\mu}_{k}<0} \frac{e^{-a\left(\mu-\tilde{\mu}_{k}\right)}}{\left(\mu-\tilde{\mu}_{k}\right) \partial_{\mu} \tilde{\varphi}\left(\lambda, \tilde{\mu}_{k} ; 0\right)}\right)
$$

The quantity $\varphi(\lambda, \mu ; 0)$ is easily evaluated in this case, and its expression is given in both subsequent subsections. 


\subsection{Positive case}

Suppose first that all the random variables $X_{n}$ are (strictly) positive: $X_{n}>0$. Then the sequence $\left(S_{n}\right)_{n \geqslant 0}$ is (strictly) increasing and $\tau_{0}<\infty$. We have

$$
\mathbb{E}\left(e^{i \mu S_{n}} \mathbb{1}_{\left\{S_{n}>0\right\}}\right)=\mathbb{E}\left(e^{i \mu S_{n}}\right)=\left[\mathbb{E}\left(e^{i \mu X}\right)\right]^{n}
$$

and formula (1) yields here

$$
\begin{aligned}
\varphi(\lambda, \mu ; 0) & =1-\exp \left(-\sum_{n=1}^{\infty} \frac{e^{-\lambda n}}{n}\left[\mathbb{E}\left(e^{i \mu X}\right)\right]^{n}\right)=1-\exp \left(-\sum_{n=1}^{\infty} \frac{\left[e^{-\lambda} \mathbb{E}\left(e^{i \mu X}\right)\right]^{n}}{n}\right) \\
& =1-\exp \left[\ln \left(1-e^{-\lambda} \mathbb{E}\left(e^{i \mu X}\right)\right)\right]=e^{-\lambda} \mathbb{E}\left(e^{i \mu X}\right) .
\end{aligned}
$$

Remark 4 In this case, the increasing times introduced in Section 2 are simply $\sigma_{n}=n$. In particular, we see that $\sigma_{1}=\tau_{0}=1$ which directly implies $\varphi(\lambda, \mu ; 0)=e^{-\lambda} \mathbb{E}\left(e^{i \mu X}\right)$.

\section{$4.2 \quad$ Non-negative case}

Suppose now that all the random variables $X_{n}$ are non-negative: $X_{n} \geqslant 0$. The sequence $\left(S_{n}\right)_{n \geqslant 0}$ is non-decreasing and $\tau_{0}<\infty$ a.s. (see Remark 5 below). We then have, setting $p=\mathbb{P}\{X=0\}$,

$$
\mathbb{P}\left\{S_{n}=0\right\}=\mathbb{P}\left\{X_{1}=\cdots=X_{n}=0\right\}=p^{n}
$$

and therefore

$$
\mathbb{E}\left(e^{i \mu S_{n}} \mathbb{1}_{\left\{S_{n}>0\right\}}\right)=\mathbb{E}\left(e^{i \mu S_{n}}\right)-\mathbb{P}\left\{S_{n}=0\right\}=\left[\mathbb{E}\left(e^{i \mu X}\right)\right]^{n}-p^{n} .
$$

As a byproduct, formula (1) yields

$$
\begin{aligned}
\varphi(\lambda, \mu ; 0) & =1-\exp \left(-\sum_{n=1}^{\infty} \frac{e^{-\lambda n}}{n}\left(\left[\mathbb{E}\left(e^{i \mu X}\right)\right]^{n}-p^{n}\right)\right) \\
& =1-\exp \left(\sum_{n=1}^{\infty} \frac{\left(p e^{-\lambda}\right)^{n}}{n}-\sum_{n=1}^{\infty} \frac{\left[e^{-\lambda} \mathbb{E}\left(e^{i \mu X}\right)\right]^{n}}{n}\right) \\
& =1-\frac{\exp \left[\ln \left(1-e^{-\lambda} \mathbb{E}\left(e^{i \mu X}\right)\right)\right]}{\exp \left[\ln \left(1-p e^{-\lambda}\right)\right]}=\frac{e^{-\lambda}\left[\mathbb{E}\left(e^{i \mu X}\right)-p\right]}{1-p e^{-\lambda}} .
\end{aligned}
$$

Remark 5 This simple result may be directly obtained. Indeed, we have for $1 \leqslant n<\infty$

$$
\mathbb{P}\left\{\tau_{0}=n\right\}=\mathbb{P}\left\{X_{1}=X_{2}=\cdots=X_{n-1}=0, X_{n}>0\right\}=p^{n-1}(1-p)
$$

from which we see that $\tau_{0}$ is geometrically distributed with parameter $1-p$ (and then $\tau_{0}<\infty$ a.s.). Moreover, $S_{\tau_{0}}=X_{1}+\cdots+X_{\tau_{0}-1}+X_{\tau_{0}}=X_{\tau_{0}}$ since $X_{1}=\cdots=X_{\tau_{0}-1}=0$. Hence

$\varphi(\lambda, \mu ; 0)=\sum_{n=1}^{\infty} e^{-\lambda n} \mathbb{E}\left(e^{i \mu X_{n}} \mathbb{1}_{\left\{\tau_{0}=n\right\}}\right)=\sum_{n=1}^{\infty} e^{-\lambda n} p^{n-1} \mathbb{E}\left(e^{i \mu X} \mathbb{1}_{\{X>0\}}\right)=\frac{e^{-\lambda} \mathbb{E}\left(e^{i \mu X} \mathbb{1}_{\{X>0\}}\right)}{1-p e^{-\lambda}}$. 


\subsection{An example: the increasing Erlangian random walk}

As an illustration of (19), we choose for the distribution of $X$ the Erlang law of parameters $\left(n_{0}, \lambda_{0}\right)$. This distribution arises often in queues theory, especially in telecommunications engineering. The density of $X$ is $\frac{\lambda_{0}^{n_{0}} x^{n_{0}-1}}{\left(n_{0}-1\right) !} e^{-\lambda_{0} x}, x>0$. Then the distribution of $S_{n}$ is the Erlang law of parameters $\left(n n_{0}, \lambda_{0}\right)$. We have

$$
\tilde{\varphi}(\lambda, \mu ; 0)=e^{-\lambda} \mathbb{E}\left(e^{-\mu X}\right)=e^{-\lambda}\left(\frac{\lambda_{0}}{\mu+\lambda_{0}}\right)^{n_{0}} .
$$

The roots of the equation $\tilde{\varphi}(\lambda, \mu ; 0)=1$ are $\tilde{\mu}_{k}=\lambda_{0}\left(\theta_{k} e^{-\lambda / n_{0}}-1\right), k \in K=\left\{0, \ldots, n_{0}-1\right\}$, where the $\theta_{k}$ 's are the $n_{0}$-th roots of 1 : $\theta_{k}=e^{i 2 k \pi / n_{0}}$. We can easily see that $\Re\left(\tilde{\mu}_{k}\right)<0$ for any $k$. Moreover, the $\mu_{k}$ 's are single roots. On the other hand, we have to check that the assumptions of Proposition 1 are fulfilled. For this, we note, with $\varphi(\lambda, \mu ; 0)=\tilde{\varphi}(\lambda,-i \mu ; 0)$, that $\lim _{R \rightarrow+\infty} e^{i \alpha R e^{i \theta}} /\left[1-\varphi\left(\lambda, \mu-R e^{i \theta} ; 0\right)\right]=0$ for $\alpha=a+\varepsilon$ and $\theta \in(0, \pi)$, or $\alpha=-\varepsilon$ and $\theta \in(-\pi, 0)$. In addition, since $\lim _{R \rightarrow+\infty}\left(\frac{\lambda_{0}}{\lambda_{0}-i\left(\mu-R e^{i \theta}\right)}\right)^{n_{0}}=0$, we can choose $R$ large enough such that

$$
\left|1-\varphi\left(\lambda, \mu-R e^{i \theta} ; 0\right)\right|=\left|1-e^{-\lambda}\left(\frac{\lambda_{0}}{\lambda_{0}-i\left(\mu-R e^{i \theta}\right)}\right)^{n_{0}}\right| \geqslant \frac{1}{2}
$$

and then, for $\alpha$ as above,

$$
\left|\frac{e^{i \alpha R e^{i \theta}}}{1-\varphi\left(\lambda, \mu-R e^{i \theta} ; 0\right)}\right| \leqslant 2 .
$$

Consequently, we can apply the dominated convergence theorem and this gives

$$
\lim _{R \rightarrow+\infty} \int_{0}^{\pi} \frac{e^{i(a+\varepsilon) R e^{i \theta}}}{1-\varphi\left(\lambda, \mu-R e^{i \theta} ; 0\right)} d \theta=\lim _{R \rightarrow+\infty} \int_{-\pi}^{0} \frac{e^{-i \varepsilon R e^{i \theta}}}{1-\varphi\left(\lambda, \mu-R e^{i \theta} ; 0\right)} d \theta=0 .
$$

Now, for evaluating the residues in (19), we need to compute

$$
\partial_{\mu} \tilde{\varphi}(\lambda, \mu ; 0)=-\frac{n_{0}}{\mu+\lambda_{0}} \tilde{\varphi}(\lambda, \mu ; 0)
$$

and then

$$
\partial_{\mu} \tilde{\varphi}\left(\lambda, \tilde{\mu}_{k} ; 0\right)=-\frac{n_{0}}{\tilde{\mu}_{k}+\lambda_{0}}=-\frac{n_{0}}{\lambda_{0} \theta_{k}} e^{\lambda / n_{0}} .
$$

Formula (19) reads here

$$
\tilde{\varphi}(\lambda, \mu ; a)=\frac{\lambda_{0}}{n_{0}} e^{-\lambda / n_{0}}\left[1-e^{-\lambda}\left(\frac{\lambda_{0}}{\mu+\lambda_{0}}\right)^{n_{0}}\right] \sum_{k \in K} \theta_{k} \frac{e^{-a\left(\mu-\tilde{\mu}_{k}\right)}}{\mu-\tilde{\mu}_{k}} .
$$

Actually, this double Laplace transform can be completely inverted. We have obtained the following explicit expression for the density of $\left(\tau_{a}, S_{\tau_{a}}\right)$.

Theorem 3 The joint distribution of the random couple $\left(\tau_{a}, S_{\tau_{a}}\right)$ is given, for $r \geqslant 1$ and $x \geqslant a, b y$

$$
\mathbb{P}\left\{\tau_{a}=r, S_{\tau_{a}} \in d x\right\} / d x=\sum_{p=1}^{n_{0}}\left(\frac{\left(a \lambda_{0}\right)^{n_{0} r-p}}{\left(n_{0} r-p\right) !} e^{-a \lambda_{0}}\right)\left(\frac{\lambda_{0}^{p}(x-a)^{p-1}}{(p-1) !} e^{-\lambda_{0}(x-a)}\right) .
$$


Proof. Observing that

$$
1-e^{-\lambda}\left(\frac{\lambda_{0}}{\mu+\lambda_{0}}\right)^{n_{0}}=\frac{\prod_{l \in K}\left(\mu-\tilde{\mu}_{l}\right)}{\left(\mu+\lambda_{0}\right)^{n_{0}}}
$$

equality (20) writes

$$
\tilde{\varphi}(\lambda, \mu ; a)=\frac{\lambda_{0}}{n_{0}} e^{-\lambda / n_{0}} e^{-a \mu} \sum_{k \in K} \theta_{k} \frac{\prod_{l \in K \backslash\{k\}}\left(\mu-\tilde{\mu}_{l}\right)}{\left(\mu+\lambda_{0}\right)^{n_{0}}} e^{a \tilde{\mu}_{k}} .
$$

Let us expand the product

$$
\prod_{l \in K \backslash\{k\}}\left(\mu-\tilde{\mu}_{l}\right)=\prod_{l \in K \backslash\{k\}}\left[\left(\mu+\lambda_{0}\right)-\left(\tilde{\mu}_{l}+\lambda_{0}\right)\right]=\sum_{p=0}^{n_{0}-1}(-1)^{n_{0}-p-1} \sigma_{n_{0}-p-1, k}\left(\mu+\lambda_{0}\right)^{p}
$$

where we set

$$
\sigma_{p, k}=\sum_{\substack{l_{1}, \ldots, l_{p} \in K \backslash\{k\} \\ l_{1}<\cdots<l_{p}}}\left(1+\frac{\tilde{\mu}_{l_{1}}}{\lambda_{0}}\right) \ldots\left(1+\frac{\tilde{\mu}_{l_{p}}}{\lambda_{0}}\right) .
$$

Referring to the expression of the roots $\tilde{\mu}_{l}$, we get $1+\frac{\tilde{\mu}_{l}}{\lambda_{0}}=\theta_{l} e^{-\lambda / n_{0}}$ and

$$
\sigma_{p, k}=e^{-p \lambda / n_{0}} \sum_{\substack{l_{1}, \ldots, l_{p} \in K \backslash\{k\} \\ l_{1}<\cdots<l_{p}}} \theta_{l_{1}} \ldots \theta_{l_{p}} .
$$

The $\sigma_{p, k}$ 's can be explicitly calculated. For this, we introduce the intermediate sums

$$
\tilde{\sigma}_{p}=\sum_{\substack{l_{1}, \ldots, l_{p} \in K \\ l_{1}<\cdots<l_{p}}} \theta_{l_{1}} \ldots \theta_{l_{p}}, \quad \tilde{\sigma}_{p, k}=\sum_{\substack{l_{1}, \ldots, l_{p} \in K \backslash\{k\} \\ l_{1}<\cdots<l_{p}}} \theta_{l_{1}} \ldots \theta_{l_{p}} .
$$

The following recursive identity can be easily checked: $\tilde{\sigma}_{p, k}=\tilde{\sigma}_{p}-\theta_{k} \tilde{\sigma}_{p-1, k}$ from which we extract

$$
\tilde{\sigma}_{p, k}=\sum_{m=0}^{p}(-1)^{m} \tilde{\sigma}_{p-m} \theta_{k}^{m}=\left(-\theta_{k}\right)^{p} \text { for } 0 \leqslant p \leqslant n_{0}-1
$$

where we use the elementary equalities: $\tilde{\sigma}_{0}=1$ and $\tilde{\sigma}_{m}=0$ for $1 \leqslant m \leqslant n_{0}-1$. Hence $\sigma_{p, k}=\left(-\theta_{k}\right)^{p} e^{-p \lambda / n_{0}}$ and then

$$
\begin{aligned}
\lambda_{0} e^{-\lambda / n_{0}} \frac{\prod_{l \in K \backslash\{k\}}\left(\mu-\tilde{\mu}_{l}\right)}{\left(\mu+\lambda_{0}\right)^{n_{0}}} & =e^{-\lambda / n_{0}} \sum_{p=1}^{n_{0}}(-1)^{p-1} \sigma_{p-1, k}\left(\frac{\lambda_{0}}{\mu+\lambda_{0}}\right)^{p} \\
& =\sum_{p=1}^{n_{0}} \theta_{k}^{p-1} e^{-p \lambda / n_{0}}\left(\frac{\lambda_{0}}{\mu+\lambda_{0}}\right)^{p} .
\end{aligned}
$$

As a byproduct, putting (23) into (22), we obtain the following expression for $\tilde{\varphi}(\lambda, \mu ; a)$ :

$$
\begin{aligned}
\tilde{\varphi}(\lambda, \mu ; a) & =\frac{1}{n_{0}} e^{-a \mu} \sum_{p=1}^{n_{0}}\left(\sum_{k \in K} \theta_{k}^{p} e^{a \tilde{\mu}_{k}}\right) e^{-p \lambda / n_{0}}\left(\frac{\lambda_{0}}{\mu+\lambda_{0}}\right)^{p} \\
& =\frac{1}{n_{0}} e^{-a \lambda_{0}} e^{-a \mu} \sum_{p=1}^{n_{0}}\left(\sum_{k \in K} \theta_{k}^{p} e^{a \lambda_{0} \theta_{k} e^{-\lambda / n_{0}}}\right) e^{-p \lambda / n_{0}}\left(\frac{\lambda_{0}}{\mu+\lambda_{0}}\right)^{p} .
\end{aligned}
$$


In view to invert the double Laplace transform $(\lambda, \mu) \longmapsto \tilde{\varphi}(\lambda, \mu ; a)$, we first expand $e^{a \lambda_{0} \theta_{k} e^{-\lambda / n_{0}}}$ into a series:

$$
\sum_{k \in K} \theta_{k}^{p} e^{a \lambda_{0} \theta_{k} e^{-\lambda / n_{0}}}=\sum_{k \in K} \theta_{k}^{p} \sum_{q=0}^{\infty} \frac{\left(a \lambda_{0} \theta_{k} e^{-\lambda / n_{0}}\right)^{q}}{q !}=\sum_{q=0}^{\infty}\left(\sum_{k \in K} \theta_{k}^{p+q}\right) \frac{\left(a \lambda_{0}\right)^{q}}{q !} e^{-\lambda q / n_{0}} .
$$

It is easily seen that the sum $\sum_{k \in K} \theta_{k}^{p+q}$ equals $n_{0}$ if $p+q$ is divisible by $n_{0}$, else vanishes. Hence, performing the change of index $q=n_{0} r-p$,

$$
\sum_{k \in K} \theta_{k}^{p} e^{a \lambda_{0} \theta_{k} e^{-\lambda / n_{0}}}=n_{0} e^{\lambda p / n_{0}} \sum_{r=1}^{\infty} \frac{\left(a \lambda_{0}\right)^{n_{0} r-p}}{\left(n_{0} r-p\right) !} e^{-\lambda r}
$$

Second, we write $e^{-a \mu}\left(\frac{\lambda_{0}}{\mu+\lambda_{0}}\right)^{p}$ as a Laplace transform, that of a shifted Erlang density:

$$
e^{-a \mu}\left(\frac{\lambda_{0}}{\mu+\lambda_{0}}\right)^{p}=\int_{a}^{\infty} e^{-\mu x}\left(\frac{\lambda_{0}^{p}(x-a)^{p-1}}{(p-1) !} e^{-\lambda_{0}(x-a)}\right) d x
$$

Finally, putting (25) and (26) into (24), it comes

$$
\tilde{\varphi}(\lambda, \mu ; a)=\sum_{r=1}^{\infty} \int_{a}^{\infty} e^{-\lambda r-\mu x} \sum_{p=1}^{n_{0}}\left(\frac{\left(a \lambda_{0}\right)^{n_{0} r-p}}{\left(n_{0} r-p\right) !} e^{-a \lambda_{0}}\right)\left(\frac{\lambda_{0}^{p}(x-a)^{p-1}}{(p-1) !} e^{-\lambda_{0}(x-a)}\right) d x
$$

from which (21) emerges.

We can deduce from (21) the marginal distributions of $\left(\tau_{a}, S_{\tau_{a}}\right)$ which are displayed in the following corollary. The density of the hitting place $S_{\tau_{a}}$ below involves an interesting family of special functions, namely the generalized hyperbolic cosines defined by

$$
\operatorname{ch}_{n, p}(z)=\sum_{q=0}^{\infty} \frac{z^{n q+p}}{(n q+p) !}, \quad n, p \geqslant 0 .
$$

Corollary 2 The distribution of $\tau_{a}$ is given, for $r \geqslant 1$, by

$$
\mathbb{P}\left\{\tau_{a}=r\right\}=\left(\sum_{s=n_{0}(r-1)}^{n_{0} r-1} \frac{\left(a \lambda_{0}\right)^{s}}{s !}\right) e^{-a \lambda_{0}}
$$

and that of $S_{\tau_{a}}$, for $x \geqslant a$, by

$$
\mathbb{P}\left\{S_{\tau_{a}} \in d x\right\} / d x=\left(\sum_{p=1}^{n_{0}} \operatorname{ch}_{n_{0}, n_{0}-p}\left(a \lambda_{0}\right) \frac{\lambda_{0}^{p}(x-a)^{p-1}}{(p-1) !}\right) e^{-\lambda_{0} x} .
$$

Proof. By integrating (21) with respect to $x$ on $[a,+\infty)$, we get

$$
\mathbb{P}\left\{\tau_{a}=r\right\}=\sum_{p=1}^{n_{0}} \frac{\left(a \lambda_{0}\right)^{n_{0} r-p}}{\left(n_{0} r-p\right) !} e^{-a \lambda_{0}}
$$

which coincides with formula (27) by performing the change of indices $p=n_{0} r-s$. Similarly, by summing (21) with respect to $r$, we get

$$
\mathbb{P}\left\{S_{\tau_{a}} \in d x\right\} / d x=\sum_{p=1}^{n_{0}}\left(\sum_{r=1}^{\infty} \frac{\left(a \lambda_{0}\right)^{n_{0} r-p}}{\left(n_{0} r-p\right) !}\right) \frac{\lambda_{0}^{p}(x-a)^{p-1}}{(p-1) !} e^{-\lambda_{0} x}
$$

which can be expressed by means of generalized hyperbolic cosines according to (28). 
Remark 6 Referring to queuing theory, we can retrieve probability (27) through anther way. Indeed, consider a queuing system where arrivals occur at Erlangian times with parameters $\left(n_{0}, \lambda_{0}\right)$. This Erlang law may be viewed as the sum of $n_{0}$ independent and exponentially distributed random variables of parameter $\lambda_{0}$. So, we can reinterpret this system as a usual Poissonian one with parameter $\lambda_{0}$ for which customers arrive according exponentially distributed inter-arrival times and for which the server treats customers by groups of $n_{0}$ persons. Reminding that the Erlang law of parameters $\left(n_{0}, \lambda_{0}\right)$ is closely connected to the Poisson law of parameter $\lambda_{0}$ according to the relationship $\mathbb{P}\left\{\operatorname{Erlang}\left(n_{0}, \lambda_{0}\right)>a\right\}=\mathbb{P}\left\{\right.$ Poisson $\left.\left(a \lambda_{0}\right)<n_{0}\right\}$, probability (27) can be written as a Poisson event:

$$
\begin{aligned}
\mathbb{P}\left\{\tau_{a}=r\right\} & =\mathbb{P}\left\{S_{r-1} \leqslant a<S_{r}\right\} \\
& =\mathbb{P}\left\{\text { Erlang }\left(n_{0} r, \lambda_{0}\right)>a\right\}-\mathbb{P}\left\{\operatorname{Erlang}\left(n_{0}(r-1), \lambda_{0}\right)>a\right\} \\
& =\mathbb{P}\left\{\text { Poisson }\left(a \lambda_{0}\right)<n_{0} r\right\}-\mathbb{P}\left\{\operatorname{Poisson}\left(a \lambda_{0}\right)<n_{0}(r-1)\right\} \\
& =\mathbb{P}\left\{n_{0}(r-1) \leqslant \operatorname{Poisson}\left(a \lambda_{0}\right) \leqslant n_{0} r-1\right\} .
\end{aligned}
$$

This is exactly (27).

Remark 7 In the particular case of an increasing exponential random walk (corresponding to the case $n_{0}=1$ ), we simply have

$\mathbb{P}\left\{\tau_{a}=r, S_{\tau_{a}} \in d x\right\} / d x=\left(\frac{\left(a \lambda_{0}\right)^{r-1}}{(r-1) !} e^{-a \lambda_{0}}\right)\left(\lambda_{0} e^{-\lambda_{0}(x-a)}\right)=\mathbb{P}\left\{\tau_{a}=r\right\} \mathbb{P}\left\{S_{\tau_{a}} \in d x\right\} / d x$.

We see that the random variables $\tau_{a}$ and $S_{\tau_{a}}$ are independent, $\tau_{a}$ being distributed like a 1-shifted Poisson law of parameter $a \lambda_{0}$ and $S_{\tau_{a}}$ like an $a$-shifted exponential law of parameter $\lambda_{0}$.

\section{Pseudo-random walk}

We finally consider the case of a pseudo-random walk. By this, we mean a sequence of pseudo-random variables $\left(X_{n}\right)_{n \geqslant 0}$ driven by a signed measure $\mathfrak{m}$ with finite variation: for any Borel set $A, \mathbb{P}\left\{X_{n} \in A\right\}=\int_{A} \mathfrak{m}(d x)$ with $\int_{-\infty}^{+\infty} \mathfrak{m}(d x)=1$, and $\int_{-\infty}^{+\infty}|\mathfrak{m}(d x)|=M<$ $+\infty$. We have $M \geqslant 1$ and if $\mathfrak{m}$ is not positive nor negative, $M>1$. Then the pseudorandom walk is defined by $S_{n}=X_{1}+\cdots+X_{n}$ and its signed distribution is defined by $\mathbb{P}\left\{S_{n} \in A\right\}=\int_{A} \mathfrak{m}^{(* n)}(d x)$ where the convolution of signed measures is classically defined by $\left(\mathfrak{m}_{1} * \mathfrak{m}_{2}\right)(A)=\int_{-\infty}^{+\infty} \mathfrak{m}_{1}(A-x) \mathfrak{m}_{2}(d x)$.

For any bounded Borel function $f$, we have

$$
\begin{aligned}
\left|\mathbb{E}\left[f\left(S_{n}\right)\right]\right| & =\left|\int \ldots \int f\left(x_{1}+\cdots+x_{n}\right) \mathfrak{m}\left(d x_{1}\right) \cdots \mathfrak{m}\left(d x_{n}\right)\right| \\
& \leqslant\|f\|_{\infty} \int_{-\infty}^{+\infty}\left|\mathfrak{m}\left(d x_{1}\right)\right| \cdots \int_{-\infty}^{+\infty}\left|\mathfrak{m}\left(d x_{n}\right)\right| \leqslant\|f\|_{\infty} M^{n} .
\end{aligned}
$$

In order the series lying in Spitzer identity be convergent, we must restrict the domain of variation of the argument $\lambda$ : we should have $\sum_{n=1}^{\infty} \frac{e^{-\lambda n}}{n}\left|\mathbb{E}\left(e^{i \mu S_{n}} \mathbb{1}_{\left\{S_{n}>0\right\}}\right)\right|<+\infty$. Due to (29), this condition is fulfilled when $M e^{-\lambda}<1$ that is $\lambda>\ln M$. In conclusion, the results of Spitzer and Nakajima hold upon assuming that $\lambda>\ln M$. This fact has been exploited by Nakajima \& Sato [4] and the author [2] when studying the first hitting time related to a continuous-time pseudo-process driven by a high-order heat-type equation. 


\section{References}

[1] Feller, W. An introduction to Probability theory and its applications, Vol. II, John Wiley \& Sons, 1971.

[2] Lachal, A. First hitting time and place for pseudo-processes driven by the equation $\frac{\partial}{\partial t}= \pm \frac{\partial^{N}}{\partial x^{N}}$ subject to a linear drift. Submitted.

[3] Nakajima, T. Joint distribution of the first hitting time and first hitting place for a random walk. Kodai Math. J. 21 (1998), 192-200.

[4] Nakajima, T. and Sato, S. On the joint distribution of the first hitting time and the first hitting place to the space-time wedge domain of a biharmonic pseudo process. Tokyo J. Math. 22 (1999), no. 2, 399-413.

[5] Widder, D.V. The Laplace transform, Princeton University Press, 1946. 